\title{
Zur Casuistik der Geburt beim kyphotischen Becken.
}

Von

Dr. R. Klien, Dresden.

Nachstehender Fall betrifft eine VII para. Die 6 früheren Geburten sind in meiner Arbeit "Die Geburt beim kyphotischen Becken", dieses Arch., Bd. 50, Heft 1 notirt (Fall 70). Bei den ersten 5 Entbindungen wurden die ausgetragenen, in Schädellage sich befindenden Kinder perforirt. Bei der 6 . Entbindung handelte es sich um Zwillinge. Das erste Kind wurde lebend in I. unvollkommener Fusslage extrahirt; Knabe, $48 \mathrm{~cm}$ lang, $2300 \mathrm{~g}$ schwer. Das zweite Kind kam in I. Steisslage; Armlösung, Extraction des Kopfes; todtes Mädchen, 47,5 cm lang, $2250 \mathrm{~g}$ schwer. Die beiden Köpfe waren gut configurabel; D. bip. 8, D. bit. 7, Per. fr.-oce. 32.

Die Maasse des lumbosacral-kyphotischen Beckens (die Körper des V. Lendenwirbels und die beiden obersten Kreuzwirbel sind zerstört) sind: Sp. 25,5 , Cr. 27,2 , Tr. 25,5 (!), C. ext. 21,5, C. d. 13,5 , D. tub. isch. 8,2 , A. G. E. 12,0 .

Wenn in der Epikrise zu dieser Entbindung gesagt wurde, dass dieselbe nur Dank der guten Configurabilität der Kindsköpfe so günstig verlaufen sei, so rechtfertigten diese Behauptung die 5 vorausgegangenen Perforationen, noch mehr aber der Verlauf der 7. Entbindung. Zu ihrem Unglück hat die Patientin, sorglos gemacht durch den günstigen Verlauf der Zwillingsgeburt, den Rath nicht befolgt, bei neu eintretender Schwangersehaft sich im 9. Lunarmonat in die Klinik behufs Einleitung der Frühgeburt zu begeben ${ }^{1}$ ).

1) Die folgende Geburtsgeschichte verdanke ich der Güte des Herrn Collegen Penzl in Starnberg. 
Am Ende einer normalen Schwangerschaft am 31. October 1896 Morgens $7 \frac{1}{2} \mathrm{~h}$ Beginn der Wehen uud Blasensprung. Am 1. Nov. von $10 \mathrm{~h}$ a. m. ab Drangwehen, sehr heftig. $31 / 4$ h p. m. plötzliches, vollständiges Auf hören der Wehen; dafür sehr heftige Schmerzen links vom Nabel und im rechten Bein. Grosses Schwächegefühl. $3 \mathrm{~h} 35$ p. m. nahm Dr. Penzl folgenden Status pr. auf: Pat. sehr bleich, kühl, fast pulslos. Das Abdomen ist in zwei sehr scharf hervortretende, kugelige Hervorwölbungen abgetheilt: Die kleinere, rechts nicht ganz bis zum Nabel reichende ist der Uterus mit Placenta; die grössere, links bis 3 Querfinger über den Nabel reichende ist das in die Bauchhöhle ausgetretene Kind. I. Schädellage. Der Kopf steht fest in der Beckenweite. Die Zange lässt sich leicht anlegen, es gelingt aber nicht, den Kopf zu extrahiren! Perforation. Cranioclasie (3 h 45 p. m.). Beim Heraustreten der Schultern erscheint eine Dünndarmschlinge, die nach vollständiger Entwickelung des Kindes reponirt wird. Blutung mässig; Massage des Uterus. Manuelle Lösung der Placenta. Dabei zeigt sich ein Riss in der linken, hinteren Wand des Uterus, der Cervix und zum Theil der Vagina. Nochmalige Reposition der Darmschlingen und Tamponade mit Jodoformgaze.

Die Entbundene ist bewusstios, Puls sehr schwach. Aether und Wein. Im Laufe des Nachmittags hebt sich der Puls etwas, das Bewusstsein kehrt zeitweise, um $7 \frac{1}{2} \mathrm{~h}$ gänzlich wieder. Um $9 \mathrm{~h}$ ist das Allgemeinbefinden ziemlich befriedigend; keine Blutung nach aussen.

2. Nov, Leib etwas anfgetrieben, weich, nicht druckempfindlich. Im Laufe des Vormittags tritt sehr häufiges Erbrechen ein, welches bis zum 4. Nov. anhält. Kein Fieber. Puls hebt sich allmählich. Die Lochien fliessen durch den Tampon gut ab; keine Blutung, kein übler Geruch.

6. Nov. Erste Entfernung der Tamponade. Tamponade der Scheide.

11. Nov. Einlauf. Stuhlgang ohne Schmerzen. findlich.

12. Nov. Stuhlgang spontan. Leib stärker aufgetrieben, etwas em-

13. Nov. Morgens $71 / 2$ h Temp. $38,8^{\circ}$ (einzige Temperatursteigerung im ganzen Verlauf der Behandlung bei täglich zweimal vorgenommener Messung). Leibschmerzen, Mattigkeit. Abends bei Entfernung der Tamponade Entleerung einer grossen, ziemlich dünnflüssigen gelblichen Kothmasse durch die Vagina. Ausspülung und neue Tamponade.

Das Allgemeinbefinden hebt sich von da ab ganz bedeutend. Täglich Ausspülung und Erneuerung der Tamponade. Zu den Lösungen wurde mit Ausnahme einiger Tage, wo ein Versuch mit Formalin gemacht wurde, welches stark reizte, ausschliesslich Lysol verwendet.

Bei einem Consilium mit den behandelnden Herren Drr. Penzl und $\mathrm{Magg}$ am 25. Nov. fand ich in dem trichterförmig in die Höhe gezogenen und fixirten Vaginalgewölbe einen fast querverlaufenden ca. $5 \mathrm{~cm}$ langen Spalt, dessen narbige Ränder auf der Grenze zwischen rechtem und mittlerem Drittel fest aneinanderlagen, hier eine sagittal verlaufende, tiefe Einziehung bildend. In dem klaffenden, rechten Drittel des Risses wulstete sich ein ca. 10 pfennigstückgrosses Stück hochrother Darmschleimhaut hervor; an dieser Stelle entleerte sich eine gelbliche, fäculente, flüssige Masse. In dem links gelegenen, zwei Drittheile einnehmenden Theil des klaffenden Spaltes wölbte sich ein etwa pflaumengrosses Stück bläulich und matter aussehender Darmschleimhaut vor. Hier trat kein Koth zu Tage. Die hintere Muttermundslippe existirte nicht mehr. Die 
Untersuchung mittelst Simon'scher Specula war sehr erschwert durch die Verengerung des Beckenausgangs. klinik.

Am 3. Dec. 96. Transferirung der Pat. in die Münchener Frauen-

Hier ergab sich bei genanerer Untersuchung, dass es sich um eine Rectovaginalfistel handelte. Man konnte nämlich ca. $10 \mathrm{~cm}$ aufwärts vom Auus mit dem Finger den Spalt in der vorderen Mastdarmwand fühlen, ferner floss in das Rectum gelassenes Spülwasser sofort per vaginam wieder ab. Unmittelbar oberhalb des genannten Spaltes stiess der untersuchende Finger sowohl als das eingeführte Spülrohr auf ein Hinderniss.

Zur Schliessung der höchst unbequem gelegenen und schwer zugänglichen Fistel wurden von Herrn Geheimrath von Winckel nacheinander 3 Operationen vorgenommen.

I. Operation am 17. Dec. 96. Zunächst wurde das links gelegene Stück prolabirter Mastdarmschleimhaut bezw. -wand, welches keine Oeffnung zeigte, ringsum stumpf von dem Vaginalsaume des Fistelrandes abgelöst in das Rectum zurückgestülpt und der angefrischte Fistelrand linear in querer Richtung durch sagittale Nähte vereinigt. Sodam wurde im rechten Drittel des ursprünglichen Vaginalrisses die prolabirte Darmschleimhaut abpräparirt und die angefrischten vaginalen Fisteliänder ebenfalls vereinigt. Nahtmaterial: Catgut.

Schon am 2. Tage geht wieder Koth per vag. ab. (8tägiges Erysipel, ausgehend von der Nase.)

II. Operation am 21. Jan. 97. Die Oeffnung im hinteren Vaginalgewölbe fast quer verlaufend, klaffe wieder auf $3-4 \mathrm{~cm}$. Ein Herabziehen der ganzen Partie sowie des Uterus ist unmöglich. Nach entsprechender Anfrischung Vermähung der vorderen Muttermundslippe mit der hinteren Vaginalwand in querer Richtung unterhalb der Oeffnung. Nahtmaterial: Catgut.

Schon am anderen Tage gehen wieder Winde, am 4. Tage Koth durch die Vagina ab.

Als III. Operation wird am 18. Febr. 97 die quere Obliteration des oberen Drittels der Vagina ausgeführt, ebenfalls mittelst Catgut. Nach 2 Tagen gehen bereits wieder Winde, am 3. Tage Koth durch die Scheide ab.

Am 28. Febr. wird Pat. anf ihren Wunsch entlassen mit dem Auftrage, sich im Mai wieder vorzustellen, welchen Rath sie aber wiederum ignorirte. Herr College Penzl theilte mir mit, dass sich der Zustand der Pat. im Verlaufe der nächsten Monate soweit gebessert habe, dass der Kothabgang durch die Scheide viel seltener wurde und hauptsächlich nur bei dünnem und breiigem Stuhl auftrat, während feste Stühle normal per anum entleert wurden. Dieser Zustand besteht bis zum heutigen Tage fort. Der Stuhlgang ist nie mit Schmerzen verbunden.

26. März 97 erste Periode; die nächsten beiden folgten nach drei Wochen, stark. Von da ab regelmässig alle 4 Wochen, schmerzlos, 2-3 Tage dauernd. Das Allgemeinbefinden ist ausgezeichnet, das Aussehen vortrefflich.

Der vorstehende Fall ist ein erneuter Beweis, . wie gefährlich der Geburtsverlauf bei kyphotischem Becken sein kann. Unglaub- 
lich beinahe erscheint die Sorglosigkeit der eindringlich verwarnten Gravida, Tadel verdient aber auch das Verhalten der Hebamme, dass sie, zumal bei der Anamnese des Falls, nicht von vornherein auf Zuziehung eines Arztes gedrungen hat. Wenn auch im Allgemeinen die Gefahr einer Uterusruptur selten eintritt, wenn der Kopf einmal im Becken steht, so gilt das naturgemäss nicht für das im Ausgang verengte Becken. Vielleicht sollte dies in den Lehrbüchern der Hebammenkunst mehr hervorgehoben werden. Auch dürfte in diesem Falle wiederum von einer besonderen Beweglichkeit der Beckengelenke keine Rede sein; bei der Zwillingsgeburt ist das Fehlen derselben ausdrücklich constatirt worden.

Für die bisher bekannten ca. 200 Geburtsfälle bei kyphotischem. Becken ist der beschriebene der 4., welcher spontan zur Uterusruptur führte; die 3 früheren (Breslau-Moor 1865, Herrgott 1877, Lambl 1858) endeten für Mutter und Kind tödtlich. Ein Fall endete tödtlich nach Kolpoaporrhexis (Neugebauer 1895). In 2 Fällen drohte die Ruptur bei im Becken stehenden Kopf; der eine (Hugenberger 1869) wurde mit der Zange beendet, die Frau starb an Sepsis, das Kind lebte; D. tub. isch. 7,2 cm. Der andere Fall wurde von Schauta (1893) symphysiotomirt; Dist. tub. 7,5 cm; Forceps, Ritgen. Ausgetragenes, lebendes Kind in Gesichtslage geboren; die Mutter genas, behielt nur eine Schwäche des Sphineter vesicae in Folge sub oper. eingetretenen Risses der Harnröhre und des Blasenhalses.

Beachtenswerth ist der Fall ferner wegen des - abgesehen von dem Bestehenbleiben der Darmfistel (s.u.) - ausgezeichneten Heilungsverlaufes der Lterusruptur dureh Gazetamponade, demjenigen Mittel, welches dem Arzte auf dem Lande fast ausschliesslich zur Verfügung steht. Ist doch auch für die Klinik die Frage noch nicht endgiltig entschieden, ob und in welchen Fällen die Operation der Tamponade vorzuziehen ist. Sehr wichtig für den Erfolg der Tamponade dürfte es aber sein, bei garnicht oder wenig gestörtem Verlaufe die erste Tamponade lange liegen zu lassen; in dem mitgetheilten Falle wurde sie erst am 6. Tage das erste Mal erneuert. Auch in der Gynäkologie mehren sich ja die Stimmen, welche ein langes Liegenlassen der ersten Tamponade empfehlen, z. B. bei der Klammerbehandlung.

Endlich ist noch mit einigen Worten der Fistel zu gedenken. Der Geburts- und Wochenbettsgeschichte nach hätte man es wohl 
für wahrscheinlich halten müssen, dass cine Dünndarmscheidenfistel entstanden wäre. Man hätte sich vorstellen können, dass sich in den Vaginalriss ein Segment der reponirten Dünndarmschlinge eingeklemmt habe, mit den Rissrändern verlöthet sei, und dass dann an dem frei in die Vagina hineinragenden Segment in Folge äusserer und bakterieller Insulte eine Perforation zu Stande gekommen wäre; damit würde der erst am 13. Tage erfolgte Abgang von Koth per vaginam übereinstimmen. Nun ergab aber die Untersuchung in der Klinik zweifellos die Thatsache, dass es sich um eine Rectovaginalfistel, und zwar nur um eine solche handelte. Hiermit stimmt wieder die Beobachtung überein, dass von Hause aus der abgehende Koth fäculent roch, kein Dünndarminhalt war. Angesichts der völligen Zerstörung der hinteren Muttermundslippe und der grossen Ausdehnung des Risses sowohl im Rectum als in der Vagina muss man daher wohl annehmen, dass der Rectovaginalriss bereits ebenfalls sub partu entstanden ist. Durch den eröfneten Douglas bezw. den Riss im Uterus prolabirte die Dünndarmschlinge. Dieser obere Riss heilte nacb Reposition der Dünndarmschlinge und Tamponade. Der untere blieb. Zur Erklärung dafür, dass der Kothabgang erst am 13. Tage erfolgte, giebt uns vielleicht einen Fingerzeig die Beschaffenheit der linken $2 / 3$ des Vaginalrisses. Hier fand sich bei der I. Operation ringsherum die Darmschleimhaut dicht verklebt mit dem vaginalen Fistelrand; hier kam kein Koth hervor. Wahrscheinlich war das anfänglich auch in dem rechten 1/3 des Risses der Fall. Zeit zu einer solchen Verklebung ist hinreichend vorhanden gewesen, da der erste Stuhlgang post partum erst am 11. Tage erfolgte. Die partielle Einstülpung der Rectalwand in den Riss, um welche es sich also offenbar gehandelt bat, musste natürlich zu einer Verengerung des Mastdarmlumens führen (vergl. oben den Widerstand, den Finger und Mastdarmrohr fanden). In Folge dieser Verengerung musste die eingestülpte Partie von der herabrückenden Kothsäule eine beträchtliche Zerrung erleiden, welche dann auch schon beim zweitfolgenden Stuhlgang, am 13. Tage, zu einem Defect der naturgemäss wenig widerstandsfähigen Verklebung mit dem Rissrande führte. Somit war von neuem eine Communication zwischen Rectum und Vagina geschaffen. Eine andere Erklärung des immerhin merkwürdigen Falles ist wohl kaum denkbar.

Von der noch immer bestehenden, theilweisen Incontinenz 
46 Klien, Zur Casuistik der Geburt beim kyphotischen Becken.

dürfte die Patientin übrigens wohl ziemlich sicher zu befreien sein, wenn sie sich zu einer ihr vorgeschlagenen nochmaligen Operation entschliessen würde.

Meinem früheren Chef, Herrn Geheimrath v. Winckel, dom verehrten Gründer und ältesten Ehrenmitglied unserer Gesellschaft, erlaube ich mir für die Ueberlassung vorstehenden Falles auch an dieser Stelle meinen besten Dank zu sagen. 